\title{
Higher harmonics and attosecond pulse generation by laser induced Thomson scattering in atomic clusters
}

\author{
Prachi Venkat" and Amol R. Holkundkar ${ }^{\dagger}$ \\ Department of Physics, Birla Institute of Technology and Science - Pilani, Rajasthan, 333031, India
}

(Received 10 May 2019; published 12 August 2019)

\begin{abstract}
The generation of higher harmonics of intense lasers and associated attosecond pulses is a field of contemporary interest which promises a variety of applications ranging from the fundamental to applied sciences. In this work, we have probed the interaction of the intense $\left(\gtrsim 10^{19} \mathrm{~W} / \mathrm{cm}^{2}\right) 248 \mathrm{~nm}$ laser with Deuterium clusters using classical molecular dynamics simulation. The Thomson scattered radiation emitted by the electrons is considered by using standard Liénard-Wiechert potentials. We have studied the angular distribution of the radiation emitted by electrons and observed that the ponderomotive force exerted by these highly intense laser pulses leaves a very distinct signature of the radiated energy along a particular direction, which in principle has its own diagnostic potential to directly measure the intensities of incident laser pulses. Furthermore, the interaction of lasers with intensities $\sim 10^{19}-10^{21} \mathrm{~W} / \mathrm{cm}^{2}$ with atomic clusters results in the attosecond burst of energy in form of electromagnetic radiations, which fall under the XUV to soft $\mathrm{x}$-rays regime of electromagnetic spectrum. The parameters of the atomic clusters, e.g., size (number of atoms), atomic species, etc. can be easily controlled experimentally and these in turn, change the number of electrons participating in the interaction process and hence, the properties of Thomson scattered radiation can be tuned accordingly.
\end{abstract}

DOI: 10.1103/PhysRevAccelBeams.22.084401

\section{INTRODUCTION}

Intense laser pulses when interact with noble gas clusters, a plasma medium of highly energetic ions and electrons is created, which finds application in numerous fields such as lithography [1], production of energetic ions [2], high harmonic generation [3] and neutron production by Deuterium fusion $[4,5]$, to name a few. The characterization of Deuterium clusters in the context of fusion experiments is reported in Ref. [6], where they have varied the experimental parameters to obtain Deuterium clusters with diameter ranging from $20-100 \AA$ for optimum neutron production. The application and utility of laser-cluster interaction may vary depending on the laser parameters and atomic species constituting the cluster. However, the basic physics behind the interaction is the initial optical field ionization of neutral atoms by the laser fields which brings electrons and ions into the picture, followed by collisional ionization of the ions which further gives rise to higher charge states. The electron cloud formed as a result

prachivenkat11@gmail.com

amol.holkundkar@pilani.bits-pilani.ac.in

Published by the American Physical Society under the terms of the Creative Commons Attribution 4.0 International license. Further distribution of this work must maintain attribution to the author(s) and the published article's title, journal citation, and DOI. of the ionization departs the cluster on account of outer ionization and the ionic cloud left behind ultimately explodes under Coulomb repulsion or hydrodynamic pressure. With the advancement in the production of highly intense laser sources such as FELs and the recent developments at ELI facility, laser fields of intensity as high as $10^{24} \mathrm{~W} / \mathrm{cm}^{2}$ can now be obtained [7]. Furthermore, high intensity $\mathrm{KrF}$ lasers of wavelength $248 \mathrm{~nm}$ are being developed at the High Intensity Laser Laboratory (HILL) [8], a unit of LaserLab, Europe [9]. Such high intensity lasers have served to expand the horizon of laser-cluster interaction. These high power lasers have assisted lot of development in the field of radiation reaction [10] and high harmonics generation by laser-plasma interaction [11,12]. Furthermore, the direct measurement of the laser parameters at such extreme laser intensities is always a challenge, which can be addressed by the knowledge of Thomson scattered radiation post electron interaction with such ultrahigh laser intensities [13].

In the classical scenario, generation of higher harmonics of the fundamental frequency of incident laser field occurs mainly due to Thomson scattering. The electrons produced during the ionization of cluster oscillate under the influence of strong laser field and emit radiation. In the relativistic scenario, when the laser strength parameter $a_{0} \gtrsim 1$, electrons experience the $\mathbf{v} \times \mathbf{B}$ heating, which significantly alters the trajectory of the electron (as compared to the cases when $a_{0} \ll 1$ ) and consequently the generated 
harmonics [14]. These radiation yields can be very well calculated by the standard Liénard-Wiechert potentials [15]. The nonlinear dynamics in ultrarelativistic scenario can cause the electrons to emit harmonics of order as high as several 10s and 100s of the fundamental frequency [16-18]. However, a beam of electrons entering a highly intense laser field can be scattered away due to the ponderomotive force of laser field before it reaches the peak of the laser field, as a consequence of which it is feasible to generate the electrons via field ionization within the laser field itself [19]. This can be achieved by the interaction of laser with an atomic cluster.

The higher order harmonics generated during lasercluster interaction can find application in the generation of terahertz radiation [20] and x-ray pulses [21,22]. The generation of higher order harmonics can be employed in a number of applications by isolating the desired harmonic from the radiation spectrum [23]. Also, this technique can be used to produce pulses of attosecond duration. Several theoretical models have been developed in the past where interaction of lasers with neutral gases has been simulated to produce higher order harmonics and desired harmonics have been selected to produce attosecond pulses through fourier synthesis $[24,25]$. This is possible because the neighboring harmonics in the radiation spectrum are locked in phase and can add constructively at a time instant to give a burst of energy of attosecond duration [26].

In this work, we have used the classical molecular dynamics simulations to study the generation of higher order harmonics by the interaction of $248 \mathrm{~nm}$, intense $\left(\gtrsim 10^{19} \mathrm{~W} / \mathrm{cm}^{2}\right), 3$ cycles laser pulses with small Deuterium clusters. We intend to explore the diagnostic potential of the radiation that is emitted during laser-cluster interaction, along with the generation of high energy photons. As a consequence of the intense laser fields, the electrons experience a drift along laser propagation direction caused by the ponderomotive force. This drift is dominated by the intensity of the laser pulse, as a result the direction in which maximum power is radiated is directly related to the peak laser intensity. Our aim is to use this property to find the typical angle of maximum radiation for a specific intensity. Furthermore, the position of the detector can be tuned to obtain the desired harmonic signal, as the intensity of a specific harmonic varies with the azimuthal and polar angles. Besides this, for different laser intensities we have also studied the generation of attosecond pulses along the direction of maximum radiated energy. The paper has been organized as follows: Sec. II entails the simulation details and the pulse model used for this study, Sec. III consists of the results of the simulations carried out for various laser-cluster parameters and Sec. IV concludes and summarizes the study.

\section{SIMULATION MODEL}

A classical molecular dynamics simulation model has been employed to study the interaction dynamics

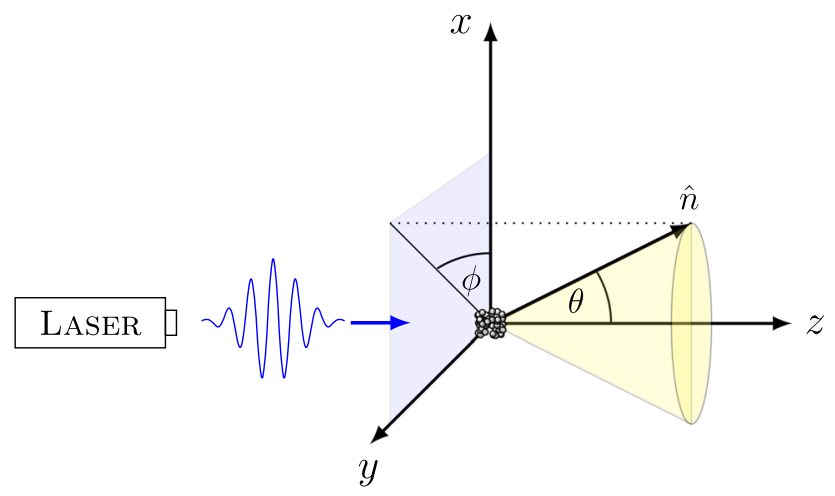

FIG. 1. Schematic diagram representing the numerical setup. The angles $\theta$ and $\phi$ are respectively measured with respect to $+z$ and $+x$ axis. The laser is incident from left on the cluster centered around origin and $\hat{n}$ points along the unit vector toward detection direction from the instantaneous position of the charged particle.

and energetics of the laser-cluster interaction [27,28]. Throughout this work we have used dimensionless units. The spatial dimension is normalized with respect to the wave vector $(k=2 \pi / \lambda)$ such that $k r \rightarrow r^{\prime}$ and time is normalized by the laser frequency $(\omega=k c)$ respectively $\left(\omega t \rightarrow t^{\prime}\right)$. The laser field amplitude is normalized as $a_{0}=|e| E / m_{e} \omega c$, where $E$ is field amplitude in SI units and $|e|$ and $m_{e}$ are electron charge and mass respectively. The laser intensity and corresponding dimensionless laser amplitude $a_{0}$ are related as: $I_{0} \lambda^{2}=1.36 \times 10^{18} a_{0}^{2}$ $\mathrm{W} \mathrm{cm}{ }^{-2} \mu \mathrm{m}^{2}$, where $I_{0}$ is the laser intensity in $\mathrm{W} \mathrm{cm}^{-2}$. The laser pulse is modeled by the standard plane wave model representing the vector potential $[29,30]$ :

$\mathbf{A}=a_{0} \exp \left(\frac{-\alpha \eta^{2}}{\tau_{0}^{2}}\right)\left[\delta \cos (\eta) \mathbf{e}_{\mathbf{x}}+\sqrt{1-\delta^{2}} \sin (\eta) \mathbf{e}_{\mathbf{y}}\right]$

where, $a_{0}$ is dimensionless laser amplitude, $\alpha \equiv 4 \ln (2)$, $\eta \equiv t-z, \tau_{0}$ is full width half maximum (FWHM) pulse duration, $\delta$ is a parameter which can be $1(1 / \sqrt{2})$ for linearly (circularly) polarized light, and $\mathbf{e}_{\mathbf{x}}$ and $\mathbf{e}_{\mathbf{y}}$ are unit vectors along $x$ and $y$ directions, respectively. The electric and magnetic field can be obtained from the vector potential as: $\mathbf{E}=-\partial \mathbf{A} / \partial t$ and $\mathbf{B}=\nabla \times \mathbf{A}$, respectively. An ultraviolet laser with $\lambda=248 \mathrm{~nm}, \tau_{0}=3$ cycles (FWHM), having the Gaussian temporal envelope of intensity varying from $5 \times 10^{19}-10^{21} \mathrm{~W} / \mathrm{cm}^{2}$ is used throughout the study and primarily, the effect of laser intensity on the interaction dynamics and consequently on the radiation spectrum is explored. The schematics of the simulation setup are depicted in Fig. 1. The clusters are considered to be centered around origin, laser pulse is propagating along the $z$ direction and the detector location can be set by specifying the angles $\theta$ (with propagation axis) and $\phi$ (with polarization axis) as represented in Fig. 1.

The number of atoms in a cluster is calculated as $N_{\text {Atoms }}=\left(R_{0} / R_{w}\right)^{3}$ [31], where $R_{0}$ is the cluster radius 
and $R_{W}$ is Wigner-Sietz radius, which depends on the species of the gas cluster under study. For the deuterium atom, $R_{W} \sim 1.73 \AA$ is considered [32]. Initially, all the atoms are randomly placed inside the cluster of radius $R_{0}$. In actual clusters, the number of atoms can be substantial due to which it would be computationally expensive to follow each ion and electron after the atoms are ionized and hence, a predefined number of macroparticles, $N_{\text {Macro }}$ is used. Each macroparticle consists of $N_{\text {Atoms }} / N_{\text {Macro }}$ of actual particles (ions or electrons), such that the chargeto-mass ratio of each particle is same as the individual actual particle. This procedure of lumping of actual particles to form pseudoparticles is similar to the one presented in Refs. [33,34]. In this paper, we have used $D_{500}$ cluster for all the simulations, and the number of macroparticles is taken to be 100 . The neutral atoms are initially ionized by the strong electric field originated from laser pulse and also by the Coulomb forces of other charged particles. Initial ionization under the effect of laser field brings electrons into the picture, which then collide with the existing ions or neutral atoms to escalate further ionization of the cluster atoms/ions. The initial ionization of cluster under the influence of laser field is carried out using Ammosov-Delone-Krainov tunnel ionization mechanism [35], and subsequently the ions undergo further ionization by colliding with the energetic electrons [36]. The details of the molecular dynamic simulations are discussed in Refs. [27,37] and hence, are not repeated here.

Following the ionization, all particles (ions + electrons) are advanced under the influence of Lorentz and Coulomb forces by solving the relativistic equations of motion [27,37]. The standard Boris leapfrog algorithm is used wherein the motion of particles in electric and magnetic fields is decomposed and $\mathbf{v} \times \mathbf{B}$ rotation is achieved correctly [38]. Boris algorithm is numerically stable and routinely used in standard particle-in-cell simulations. A separate module has been included in the existing MD code to study the radiation emitted by an accelerated charged particle. After calculating the trajectory of the particle $[\mathbf{r}(t), \mathbf{v}(t), \dot{\mathbf{v}}(t)]$ The energy radiated per unit solid angle per unit frequency can be calculated by Liénard-Wiechert potentials and in normalized units is given by $[15,39]$ :

$$
\begin{aligned}
\frac{d^{2} I}{d \omega d \Omega}= & \sum_{k} \mid \int_{-\infty}^{\infty} \frac{\hat{n}_{k} \times\left[\left(\hat{n}_{k}-\vec{\beta}_{k}\right) \times \overrightarrow{\dot{\beta}}_{k}\right]}{\left(1-\vec{\beta}_{k} \cdot \hat{n}_{k}\right)^{2}} \\
& \times\left.\exp \left\{i\left[t+R_{k}(t)\right] s\right\} d t\right|^{2}
\end{aligned}
$$

where, $\vec{\beta}_{k} \equiv \mathbf{v} / c$ and $\overrightarrow{\dot{\beta}}_{k} \equiv \dot{\mathbf{v}}_{\mathbf{k}} / c$ are the velocity and acceleration of $k$ th charged particle (either electron or ion) as evaluated at the retarded time, $R_{k}$ is the distance from charged particle to the observation point, and $\hat{n}_{k}$ is the unit vector from the $k$ th charged particle to the direction of detector located far away. The radiation signal is then summed over all the electrons and ions (Though ionic motion is negligible on the timescales of the laser pulse, it is included here.). The laser pulse is polarized along the $x$ direction, the angles $\theta$ and $\phi$ can be spanned to cover the space where the radiation will be eventually detected [refer Fig. 1]. The radiation spectrum is evaluated for a given $\theta$ and $\phi$. The spectrum evaluates the emitted radiation's intensity and frequency as a multiple of $s \equiv \omega / \omega_{o}$, which is basically is the harmonic of the fundamental frequency. Furthermore, the total power radiated per unit solid angle is given by [15]:

$\overrightarrow{\mathcal{A}}_{k}(t)=\frac{\hat{n}_{k} \times\left[\left(\hat{n}_{k}-\vec{\beta}_{k}\right) \times \overrightarrow{\dot{\beta}}_{k}\right]}{\left(1-\vec{\beta}_{k} \cdot \hat{n}_{k}\right)^{3}} \Rightarrow \frac{d P(t)}{d \Omega}=\sum_{k}\left|\overrightarrow{\mathcal{A}}_{k}(t)\right|^{2}$

and hence the energy radiated per unit solid angle can be calculated as:

$$
\mathcal{E}_{\text {rad }}=\int_{-\infty}^{\infty} \frac{d P(t)}{d \Omega} d t
$$

In a typical rf cavity based linear accelerator, or for that matter laser wakefield based electron acceleration schemes, the quality of the electron beam is of paramount importance and is measured in terms of the beam divergence and emittance. However, in this work we are dealing with the electrons originated from a cluster of say 500 atoms or so, and as a result this bunch of electrons collectively wiggles in the laser field. The spacecharge effect of the cluster electrons is negligible as compared to the strong laser field, which results in lower emittance and lower divergence of the electron bunch [37]. In the following section we discuss our main results on cluster dynamics, angular distribution of the emitted radiation and their respective spectral distribution.

\section{RESULTS AND DISCUSSION}

We study the interaction of the $248 \mathrm{~nm}, 3$ cycles, Gaussian laser pulse having peak intensity of $10^{20} \mathrm{~W} / \mathrm{cm}^{2}\left(a_{0} \sim 2.12\right)$ with deuterium $\left(D_{500}\right)$ cluster having the radius of $\sim 13.5 \AA$ (average distance of the ions from the cluster center is treated as cluster radius or cluster boundary). The laser is considered to be polarized along $x$ (and $y$ for circular polarization) direction. In Fig. 2, we present the temporal evolution of average kinetic energy of the electrons, inner electrons (ionized electrons inside the cluster boundary), outer electrons (electrons outside the cluster boundary), temporal pulse profile at the origin and components of total acceleration of all the electrons for linearly and circularly polarized pulses. It can be seen in Figs. 2(a) and 2(d) that the cluster gets fully ionized at the leading edge of the laser pulse. The outer 

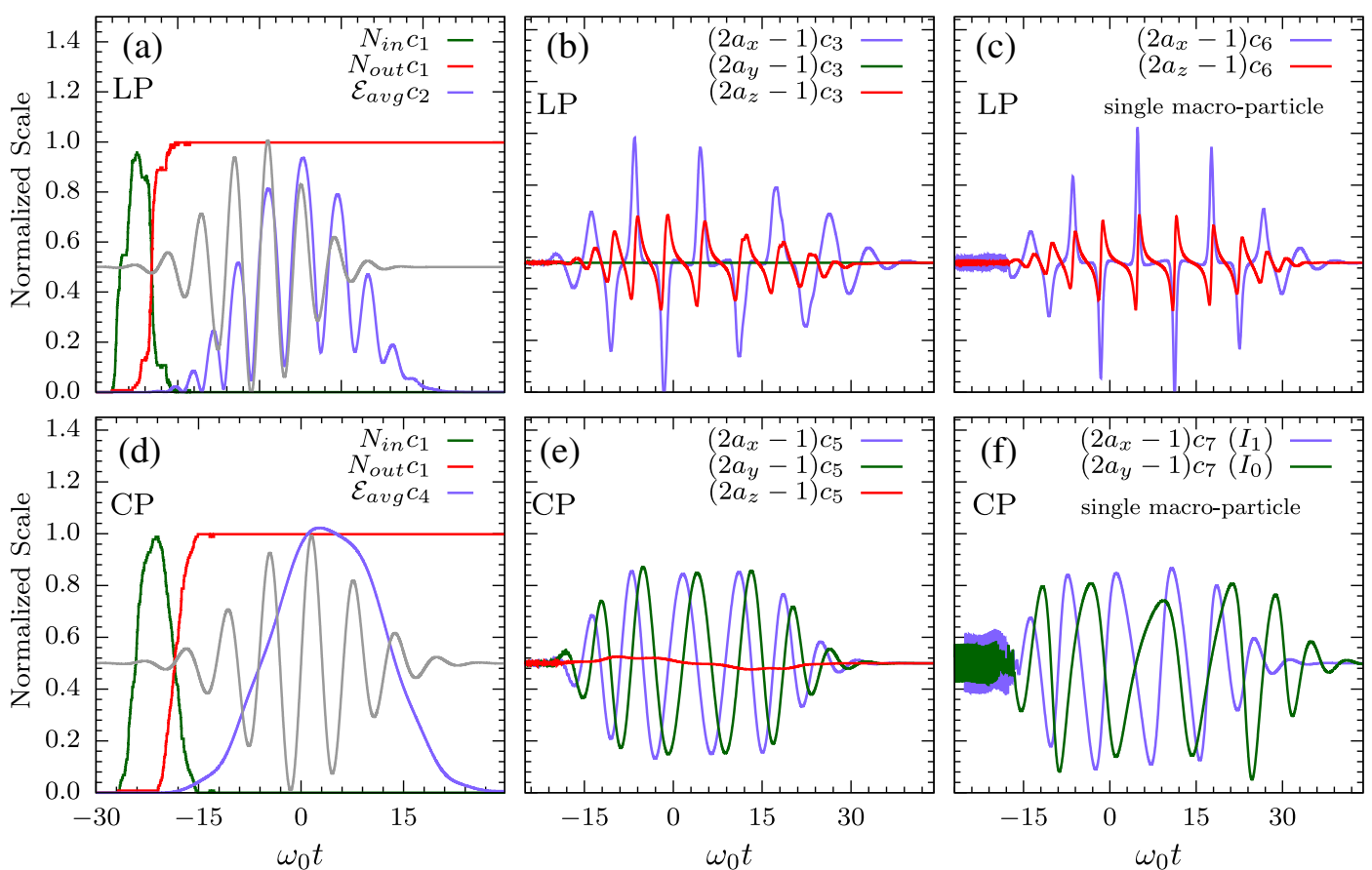

FIG. 2. The temporal evolution of the number of inner $\left(N_{\text {in }}\right)$ and outer $\left(N_{\text {out }}\right)$ electrons, average kinetic energy of the electrons $\left(\mathcal{E}_{\text {avg }}\right)$, total electron acceleration $\left(a_{x}, a_{y}, a_{z}\right)$ are presented for linearly (circularly) polarized light in top (bottom) row. All the plots are presented on a normalized scale ( 0 to 1$)$ with following scaled dimensionless constants: $c_{1}=500, c_{2}=1.2 \mathrm{MeV}, c_{3}=120$, $c_{4}=0.166 \mathrm{MeV}, c_{5}=62, c_{6}=2$, and $c_{7}=0.6$. We have also presented the acceleration for single macroparticle in (c) and (f). In order to have the same energy content in the laser pulse, for $C P$ pulse the laser intensity is considered to be $0.5 I_{0}$ (e). We have also compared the effect of the same laser intensity $\left(I_{0}\right)$ and reduced laser intensity $\left(I_{1} \equiv 0.5 I_{0}\right)$ on the acceleration for single macro-particle case (f). The temporal profile of the laser pulse, as evaluated at origin $x=y=z=0$ is also illustrated (in grey color) for reference. Please note that the normalized acceleration (ranging from -1 to 1 ) in (b), (c), (e), and (f) is mapped to the range 0 to 1 . Acceleration at any instance can be calculated using $(2 a-1) c_{3}$, say at $\omega_{0} t=4.6, x$ component of the dimensionless electron acceleration is $[2(0.94)-1] 120=105.6$

ionization of the cluster is represented by the number of outer electrons (the red curve) and it is seen that the ions are stripped of their electrons way before the pulse amplitude peaks. These electrons now respond to the oscillating electromagnetic fields as prescribed by the laser pulse envelope. Temporal profile of the total electron acceleration (summed over all the electrons) is also illustrated in Figs. 2(b) and 2(e). For linearly polarized pulse we can clearly see quite a prominent acceleration along the $z$ direction (laser propagation direction) [refer Fig. 2(b)]. However, for circularly polarized pulse it is comparatively small [refer Fig. 2(e)]. This oscillatory nature of the acceleration along the $z$ direction in case of linearly polarized pulses is the manifestation of $\mathbf{v} \times \mathbf{B}$ heating of electrons, which is completely suppressed for circularly polarized pulses. The suppression of the $\mathbf{v} \times \mathbf{B}$ heating for circularly polarized pulse is because the net electric field component continuously rotates in $x-y$ plane and so does the magnetic field component and as a result, we have the $x$ and $y$ components of acceleration [refer Fig. 2(e)]. Due to this, the radiation emitted by the electrons will be same in all azimuthal directions $(\phi)$. Thus, in order to select a particular harmonic for further application, linearly polarized pulse is preferred for laser-cluster interaction. In the following study, we will stick with the linearly polarized laser pulses only. We have also presented the acceleration component for the case when we have just a single macroparticle present, having the same charge to mass ratio. It can be seen that acceleration in all the directions [refer Fig. 2(c)] clearly resembles the total acceleration when cluster is modeled using 100 macroparticles [refer Fig. 2(b)]. Similarly, for circularly polarized pulses also, one can see the resemblance of $x$ component of acceleration among the single [refer Fig. 2(f)] and multi macroparticle cases [refer Fig. 2(e)]. We have also presented the $y$ component of acceleration of a single macroparticle in Fig. 2(f) using the same intensity for circularly polarized pulse as used for linearly polarized pulse, and a distortion in the particle trajectory is witnessed because of the enhanced field strengths in both the polarization directions. Thus, we can infer from the comparison of acceleration components of single and multi macroparticles cases that all the constituent electron macroparticles oscillate in the same manner in the laser field and hence the space-charge effect can be ignored for such strong laser fields. Moreover, for single macroparticle case in Figs. 2(c) and 2(f), we do see some small oscillations in the electron acceleration at the beginning of 


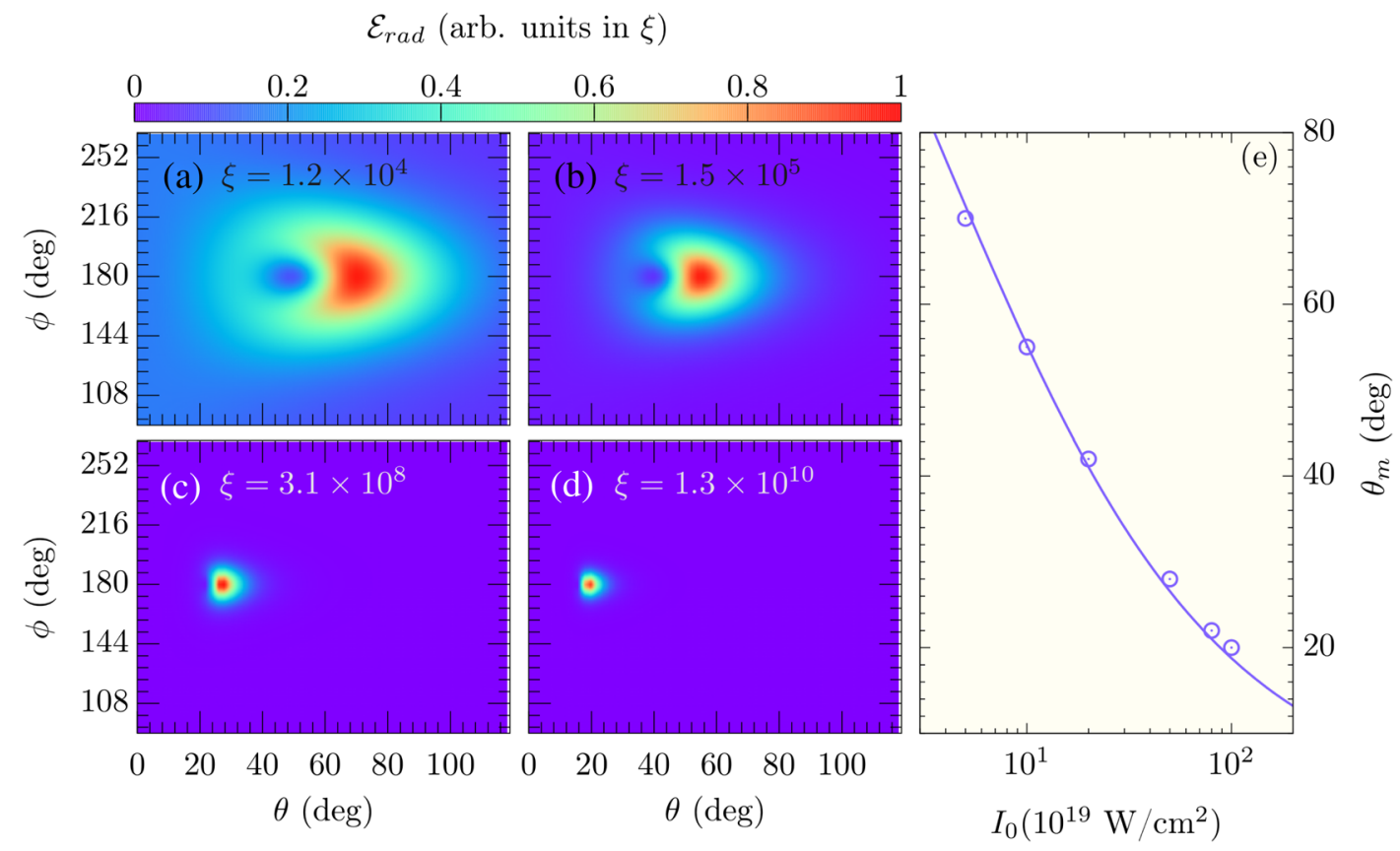

FIG. 3. Normalized angular distribution (scale factor $\xi$ ) of the total energy radiated per unit solid angle $\left(\mathcal{E}_{\text {rad }}\right)$ is presented for (a) $I=5 \times 10^{19} \mathrm{~W} / \mathrm{cm}^{2}$, (b) $10^{20} \mathrm{~W} / \mathrm{cm}^{2}$, (c), $5 \times 10^{20} \mathrm{~W} / \mathrm{cm}^{2}$ and (d) $10^{21} \mathrm{~W} / \mathrm{cm}^{2}$. The angle at which maximum energy is detected $\left(\theta_{m}\right)$ vs laser intensity is presented as open circles in (e), and solid line represents the theoretical estimate as given by Eq. (19).

the pulse. These small oscillations in the acceleration are mainly because of the effect of single ion and single electron macroparticle. The atom is ionized by the leading edge of the pulse, and is basically responding to the Coulombic force of the parent ion. However, for large number of macroparticles this Coulombic force is averaged out to be zero, and we do not see any such oscillations in Figs. 2(b) and 2(e).

Next, we consider the angular distribution $(\theta-\phi$ plane $)$ of the total energy radiated per unit solid angle $\left(\mathcal{E}_{\text {rad }}\right)$ for different laser intensities, and the results are presented in Fig. 3. The angle at which the maximum energy will be emitted is also plotted for different laser intensities. It can be seen from the Fig. 3, that the energy radiated per unit solid angle is centered around $\phi=180^{\circ}$ (and also around $\phi=0^{\circ}$ or $360^{\circ}$ ). This is because the laser is polarized along the $x$ direction, and hence $\phi=0^{\circ}, 180^{\circ}, 360^{\circ}$ are actually the directions in which the oscillating electron (along the laser field) is predominantly emitting the radiation. The signals detected at $\phi=180^{\circ}$ and $\phi=0^{\circ}$ are exactly identical but we have chosen to present the signal around $\phi=180^{\circ}$. Furthermore, as can be observed the signal shifts toward lower values of $\theta$ as we increase the laser intensity, and also the angular width reduces with the increasing laser intensity. This feature can be explained by the increased ponderomotive push along the direction of laser propagation, in addition to the angle at which maximum energy is radiated. The ponderomotive push is directly related to the peak laser intensity, as a result the angular distribution presented in the Fig. 3 can serve as a diagnostic tool to measure the peak laser intensity. In view of this, we also present our quantitative estimates for the angle at which maximum energy will be emitted/detected $\left(\theta_{m}\right)$.

The trajectory of an electron under the influence of a very intense laser field can be obtained by solving the relativistic equations of motion [40,41]:

$$
\frac{d \mathbf{P}}{d t}=q[\mathbf{E}+\mathbf{v} \times \mathbf{B}]
$$

where, $\mathbf{P}=\gamma m_{e} \mathbf{v}$ is the relativistic momentum, $\mathbf{E}$ and $\mathbf{B}$ are the electromagnetic fields associated with the laser pulse, $q$ and $m_{e}$ are the charge and mass of the electron. If the laser is polarized along $x$ direction $\left(\mathbf{E}=E_{x} \hat{x} ; \mathbf{B}=B_{y} \hat{y}\right.$ and $B_{y}=E_{x} / c$ ), then the dynamics along $x$ and $z$ directions is governed by:

$$
\begin{gathered}
\frac{d\left(\gamma v_{x}\right)}{d t}=\frac{q}{m_{e}}\left(E_{x}-v_{z} B_{y}\right)=\frac{q E_{x}}{m_{e}}\left(1-\frac{v_{z}}{c}\right) \\
\frac{d\left(\gamma v_{z}\right)}{d t}=\frac{q}{m_{e}} v_{x} B_{y}=\frac{q E_{x}}{m_{e}} \frac{v_{x}}{c} .
\end{gathered}
$$

The rate change of the energy is given by:

$$
\frac{d \gamma}{d t}=\frac{q}{m_{e} c^{2}}(\mathbf{E} \cdot \mathbf{v})=\frac{q}{m_{e} c^{2}} E_{x} v_{x} .
$$

From, Eqs. (7) and (8): 


$$
\frac{d\left(\gamma v_{z}\right)}{d t}=c \frac{d \gamma}{d t} \Rightarrow \frac{v_{z}}{c}=1-\frac{1}{\gamma}
$$

and from Eqs. (6), (8), and (9) we have:

$$
\begin{gathered}
\frac{d\left(\gamma v_{x}\right)}{d t}=\frac{q E_{x}}{m_{e}} \frac{1}{\gamma}=\frac{c^{2}}{\gamma v_{x}} \frac{d \gamma}{d t} \\
\left(\gamma v_{x}\right) \frac{d\left(\gamma v_{x}\right)}{d t}=c^{2} \frac{d \gamma}{d t} \Rightarrow \frac{d}{d t}\left(\gamma^{2} v_{x}^{2}-2 \gamma c^{2}\right)=0
\end{gathered}
$$

by using the initial conditions, i.e., $\gamma=1$ for $v_{x}=0$ we can easily obtain the expression for the $v_{x}$ as:

$$
\frac{v_{x}}{c}=\frac{\sqrt{2(\gamma-1)}}{\gamma} .
$$

Furthermore, the relativistic factor $\gamma$ can also be represented as a function of the laser field amplitude as:

$\frac{d P_{x}}{d t}=q E_{x}-q v_{z} B_{y}=-q \frac{d A_{x}}{d t} \Rightarrow P_{x}=\gamma m_{e} v_{x}=-q A_{x}$

where, $\mathbf{A}$ is the vector potential associated with the electromagnetic fields of the laser $(\mathbf{E}=-\partial \mathbf{A} / \partial t ; \mathbf{B}=$ $\nabla \times \mathbf{A})$. Using Eqs. (12) and (13) we can obtain the expression for relativistic factor as:

$$
\gamma=1+\frac{q^{2} A_{x}^{2}}{2 m_{e}^{2} c^{2}} \equiv 1+\frac{a_{0}^{2}}{2}
$$

here, $a_{0}$ is the dimensionless laser amplitude defined previously. From Eqs. (12), (9), and (14), the velocity components then can be written as:

$$
\frac{v_{x}}{c}=\frac{2 a_{0}}{2+a_{0}^{2}} ; \quad \frac{v_{z}}{c}=\frac{a_{0}^{2}}{2+a_{0}^{2}} .
$$

The strong ponderomotive push of laser along the propagation direction can easily be estimated by Eq. (15) in terms of the angle $\left(\theta_{\text {ponder }}\right)$ it makes with the $z$ axis [40]:

$$
\theta_{\text {ponder }}=\tan ^{-1}\left(\frac{v_{x}}{v_{z}}\right)=\tan ^{-1}\left(\frac{2}{a_{0}}\right) .
$$

The angle at which maximum radiation $\left(\theta_{l w}\right)$ is emitted when velocity and acceleration are along the same direction can be estimated by the Liénard-Wiechert potentials [15]:

$$
\theta_{l w}=\cos ^{-1}\left(\frac{\sqrt{1+15 \beta^{2}}-1}{3 \beta}\right)
$$

here,

$$
\beta \equiv \sqrt{1-\frac{1}{\gamma^{2}}}=\sqrt{1-\frac{1}{\left(1+0.5 a_{0}^{2}\right)^{2}}}
$$

is the velocity of charged particle and $\theta_{l w}$ is the angle it makes with the velocity vector. Now, from Eq. (16) we can estimate the direction of electron motion with respect to the propagation axis, and Eq. (17) would predict the angle (with respect to the velocity vector) at which maximum radiation is emitted and as a result, the estimated angle at which maximum radiation is emitted is given by:

$$
\begin{gathered}
\theta_{m}=\theta_{\text {ponder }}+\theta_{l w} \\
\theta_{m}=\tan ^{-1}\left(\frac{2}{a_{0}}\right)+\cos ^{-1}\left(\frac{\sqrt{1+15 \beta^{2}}-1}{3 \beta}\right) .
\end{gathered}
$$

We observe from the Fig. 3 that for all intensities the maximum energy is radiated along the direction $\phi=180^{\circ}$ and $\theta=\theta_{m}$. For circularly polarized pulses the harmonics would be independent of $\phi$, because of the constant rotation of laser field components. However, the linearly polarized laser pulse would cause the angular dependence $(\phi)$ of radiation spectra [refer Fig. 1]. In view of this, we calculate the radiation spectra for different laser intensities by varying the angle $\phi$, and the results are presented in Fig. 4. The spectrum is calculated for $\phi=0-180^{\circ}$, with fixed $\theta=90^{\circ}$ and $\theta=\theta_{m}$ associated with respective laser intensity [refer Fig. 3(e)]. It can be clearly seen from this figure that the intensity of harmonics and number of harmonics obtained are higher for the cases where $\theta=\theta_{m}$. Furthermore, while discrete harmonics are observed for the lower intensities along different $\phi$ angles [Fig. 4(a)], for higher intensities the discrete harmonics are washed out and we obtain a continuous spectrum [Fig. 4(c)]. Also, the spectrum obtained is slightly asymmetric in $\phi$, which is mainly due to the small duration (3 cycles) of the laser pulse. In our studies we have always used the carrierenvelope-phase (CEP) to be 0 radians, which might lead to the asymmetric laser field configuration at the leading and trailing edge of the laser pulse. Changing the CEP might slightly change the harmonic spectrum as well. However, changing CEP for longer pulses would not affect the laser pulse drastically and the electrons will tend to emit equivalently in both the directions, and hence the spectrum is expected to symmetric in $\phi$.

In Fig. 5, we present the frequency spectrum as evaluated for $\phi=180^{\circ}$ and $\theta=\theta_{m}$ for different laser intensities. We have also calculated the frequency integrated energy per unit solid angle as detected at $\theta=\theta_{m}$ and $\theta=90^{\circ}$. It can be seen that the energy radiated along $\theta_{m}$ is higher as compared to that detected along the $90^{\circ}$. This is mainly because of the ponderomotive push of the laser which bends the electron trajectory toward the laser propagation direction, as a result of which more energetic radiation is detected along $\theta_{m}$. The generation of the radiations having 

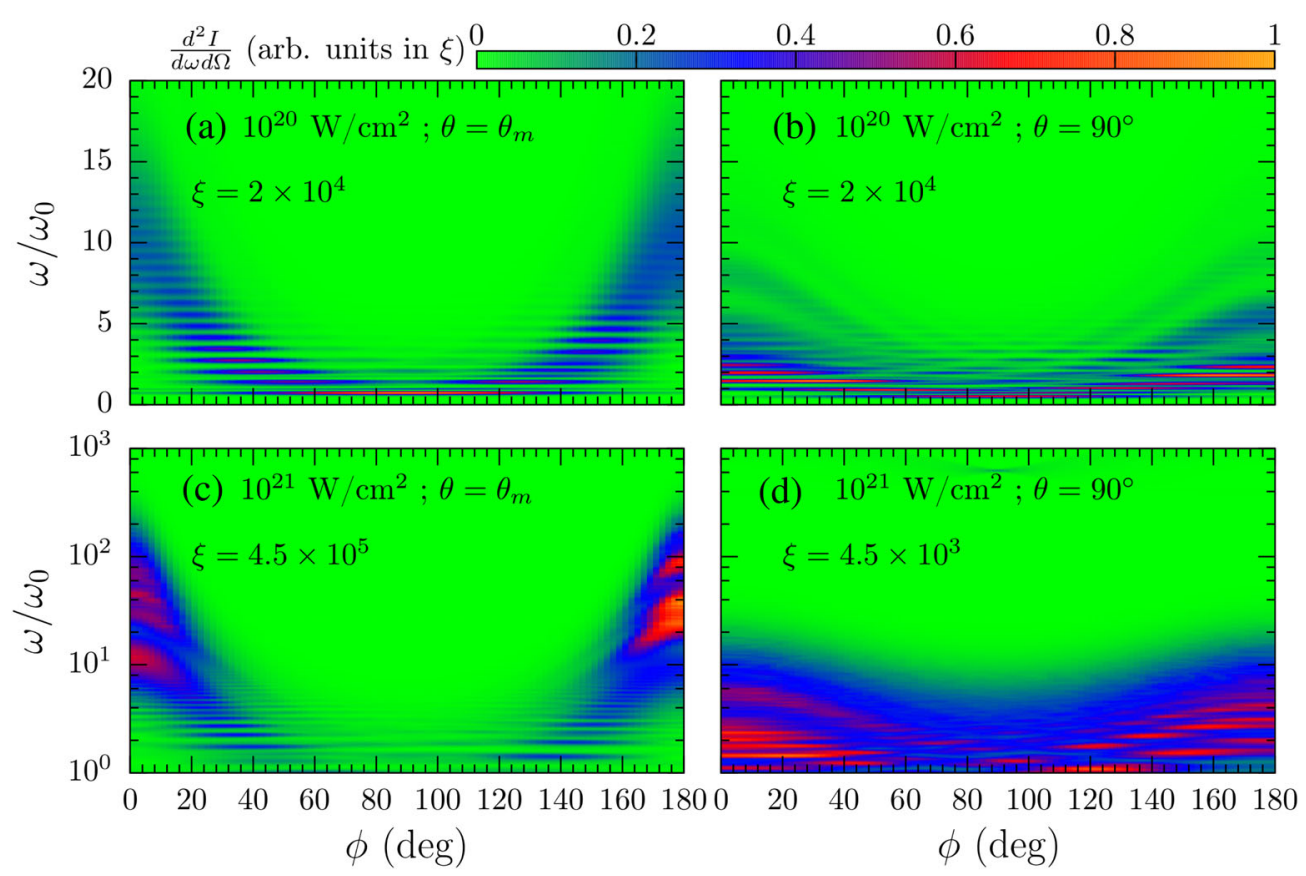

FIG. 4. The angular distribution of different harmonics is presented for $10^{20} \mathrm{~W} / \mathrm{cm}^{2}$ and $10^{21} \mathrm{~W} / \mathrm{cm}^{2}$, with $\phi$ varying from 0 to $180^{\circ}$ for a fixed $\theta=\theta_{m}$ and $\theta=90^{\circ}$. The respective scaling factors are also shown in the plots.

photon energies $\sim 25 \mathrm{eV}-465 \mathrm{eV}$ (XUV to soft x-ray) are observed ( $\hbar \omega_{0} \sim 5 \mathrm{eV}$ for $248 \mathrm{~nm}$ laser) respectively for the intensities ranging from $5 \times 10^{19}-10^{21} \mathrm{~W} / \mathrm{cm}^{2}$ [refer Fig. 5]. We have summarized all the physical observable quantities for different laser intensities in Table I.
The harmonics generation by the laser-cluster interaction can be a source of highly energetic bursts of attosecond pulses as well [42]. There are various methods to produce attosecond pulses such as Fourier synthesis [43] and stimulated Raman scattering [44]. However, it has
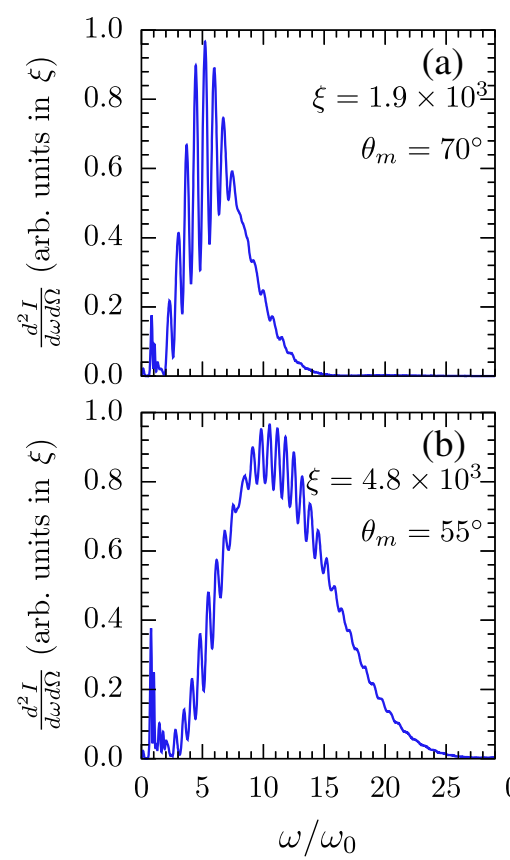

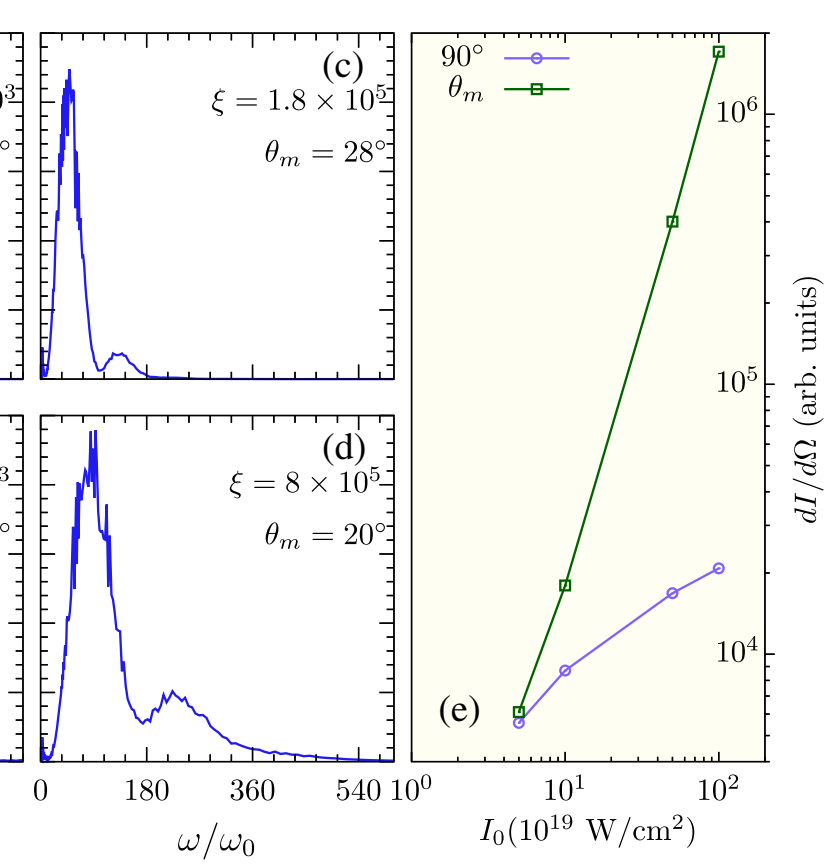

FIG. 5. Radiation spectra emitted by the electrons have been plotted for $I=5 \times 10^{19} \mathrm{~W} / \mathrm{cm}^{2}(\mathrm{a}), 1 \times 10^{20} \mathrm{~W} / \mathrm{cm}^{2}(\mathrm{~b}), 5 \times$ $10^{20} \mathrm{~W} / \mathrm{cm}^{2}(\mathrm{c})$ and $1 \times 10^{21} \mathrm{~W} / \mathrm{cm}^{2}(\mathrm{~d})$. The frequency integrated energy per unit solid angle $(d I / d \Omega)$ for different intensities of laser pulse is also plotted (e). The radiation spectra in (a),(b),(c), and (d) are calculated for $\theta=\theta_{m}$ and $\phi=180^{\circ}$. However, $d I / d \Omega$ in (e) is presented for both $\theta=90^{\circ}$ and $\theta_{m}$. 
TABLE I. Summary of physical observables determined by using different laser intensities. Table highlights the angles at which maximum energy is radiated $\left(\theta_{m}\right)$, the most prominent harmonics emitted along $\theta_{m}$ and the duration of the associated attosecond pulse.

\begin{tabular}{lccc}
\hline \hline$I_{0}\left[\mathrm{~W} / \mathrm{cm}^{2}\right]$ & $\theta_{m}$ & {$\left[\omega / \omega_{0}\right]_{\text {peak }}$} & $\tau_{\text {as }}[$ as $]$ \\
\hline $5 \times 10^{19}$ & $70^{\circ}$ & $5(\sim 25 \mathrm{eV})[\mathrm{XUV}]$ & 80 \\
$1 \times 10^{20}$ & $55^{\circ}$ & $11(\sim 55 \mathrm{eV})[\mathrm{XUV}]$ & 91 \\
$5 \times 10^{20}$ & $28^{\circ}$ & $49(\sim 245 \mathrm{eV})[$ soft X-ray] & 182 \\
$1 \times 10^{21}$ & $20^{\circ}$ & $93(\sim 465 \mathrm{eV})[$ soft X-ray] & 276 \\
\hline \hline
\end{tabular}

been observed that higher order harmonics generation has the maximum potential to produce a single attosecond pulse instead of a pulse train [40]. Relativistic nonlinear Thomson scattering is also used to produce higher order harmonics, which in turn lead to the generation of single attosecond pulses $[45,46]$. High energy bursts of attosecond duration are mainly produced by the locking of adjacent harmonics in phase when they interfere constructively $[26,40]$. For this process, it is necessary that the electrons emit the radiations in a coherent manner. This can be ensured if the radiation from different electrons reaches the detector in such a way that the travel time is comparable to or less than the attosecond pulse width produced by a single electron radiation [47]. For typical clusters of few thousands of atoms, this dimension would be of the order of few nanometers, which a signal emitted by each electron in the cluster can travel in tens of attoseconds.

We have calculated the intensity and duration of attosecond bursts of energy from the electrons by using Eq. (3). The emitted signal profile is presented in Fig. 6 for different laser intensities. The signals are detected along the direction of respective $\theta_{m}$ [refer Table I]. The attosecond pulse duration for different intensities have also been summarized in Table I. It has been observed that as we increase the laser intensity the pulse duration increases, and also the peak frequency of the emitted radiation. This looks a bit counterintuitive, however in several of the past studies, intense laser interaction with counterpropagating relativistic electron bunches $\left(\gamma_{0} \gtrsim 100\right)$ has been used as a source to generate the attosecond pulses. In this kind of backscattered Thomson radiation, the pulses of few tens of the attosecond and even zeptoseconds are reported [48], mainly caused by the relativistic Doppler effect [49]. However, in the present work, the laser first ionizes a neutral cluster to produce the seed electrons for radiation. Since a large number of ions are also involved in this interaction, Coulombic forces are also experienced by the electrons. Also, in this case the laser is effectively interacting with electrons at rest as opposed to a counterpropagating laser-electron beam interaction, and most importantly the ponderomotive force of the laser drags the electrons along with the pulse, and as a result the electrons comove with the laser pulse, experiencing the acceleration for a longer duration. As a consequence of these factors, we
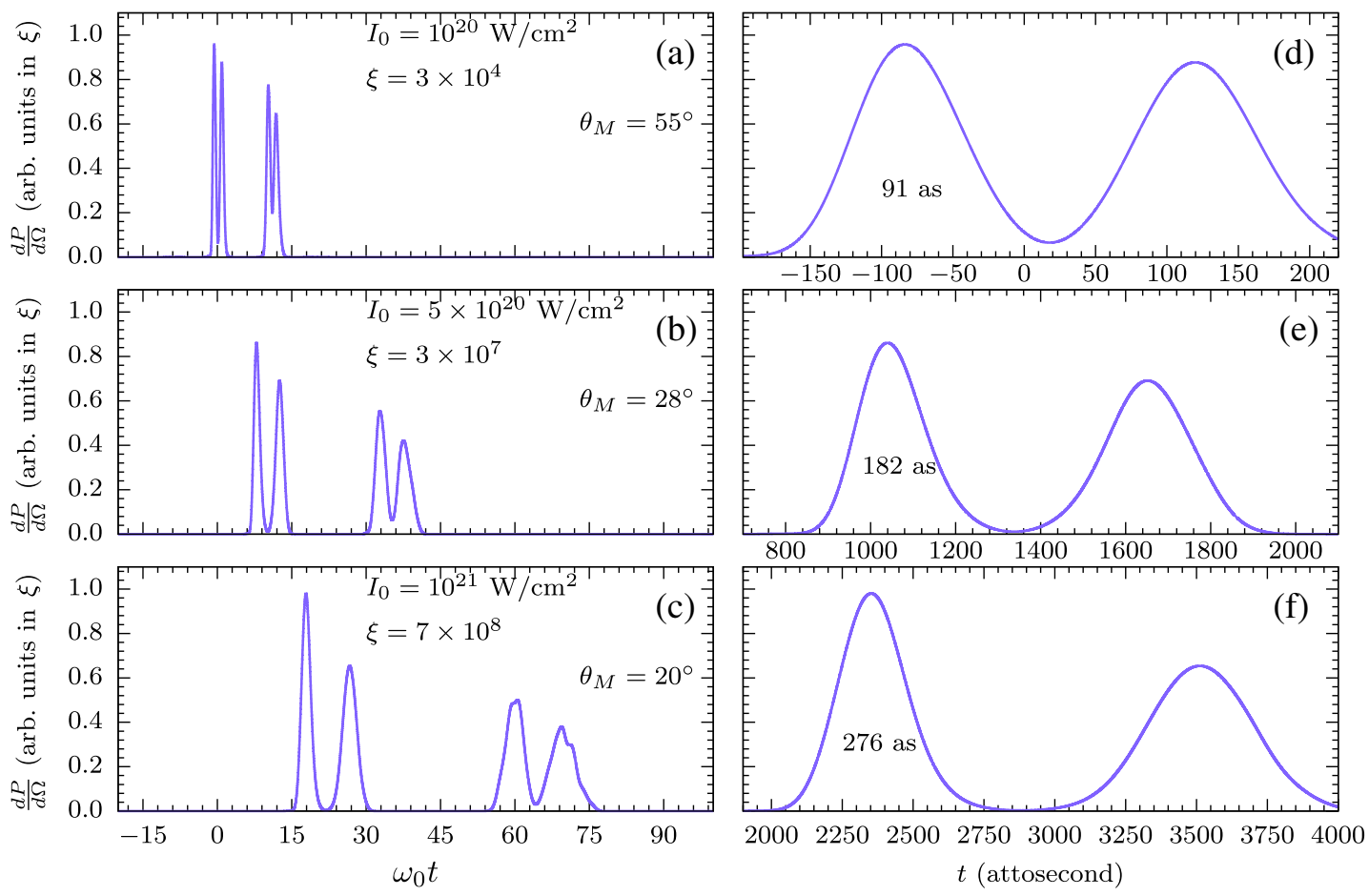

FIG. 6. The attosecond burst of the radiated energy has been presented for the intensities (a) $10^{20} \mathrm{~W} / \mathrm{cm}^{2}$, (b) $5 \times 10^{20} \mathrm{~W} / \mathrm{cm}^{2}$, and (c) $10^{21} \mathrm{~W} / \mathrm{cm}^{2}$ along the direction of respective $\theta_{m}$. The zoomed signal has been also shown in panels (d)-(f). 

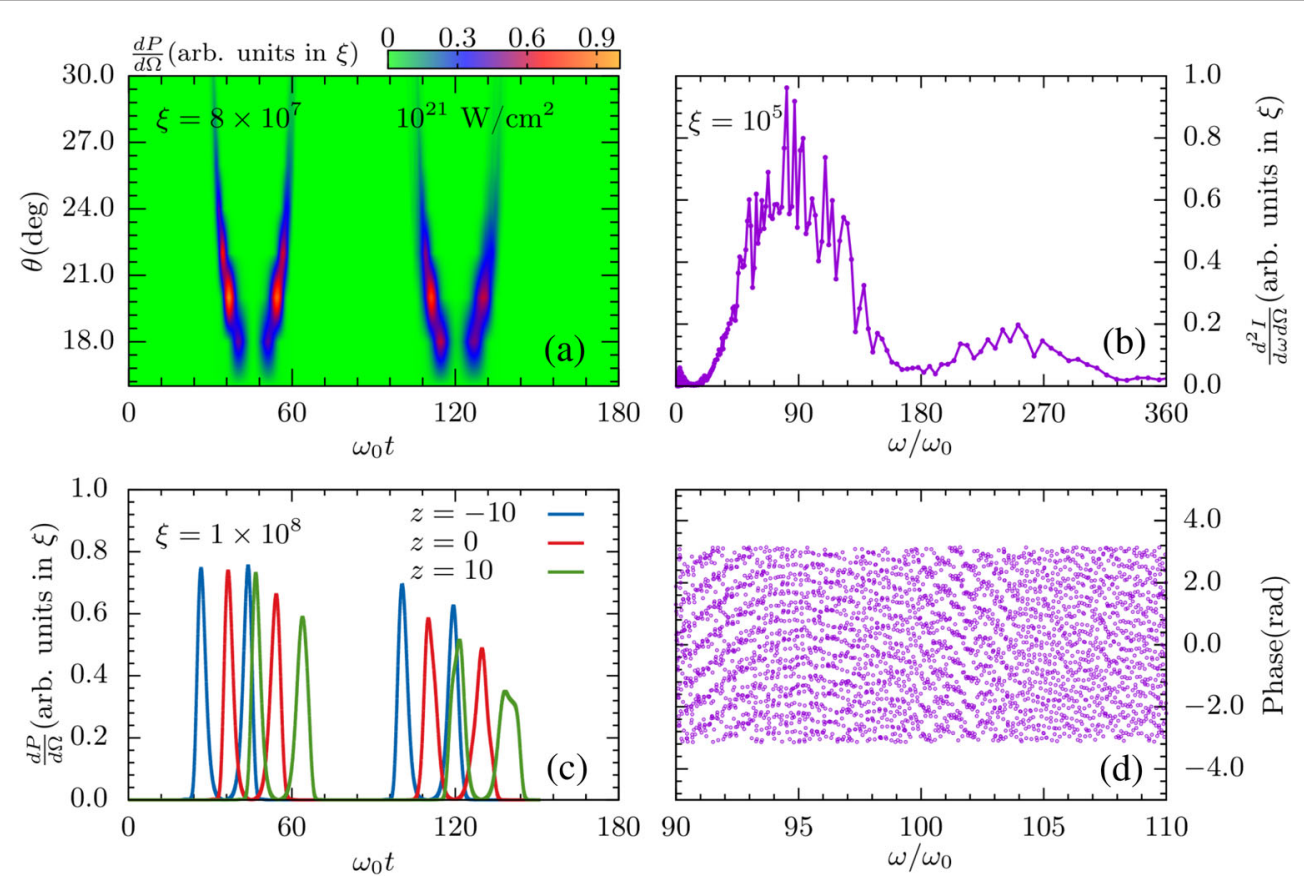

FIG. 7. The emitted attosecond pulses along different $\theta$ direction is corroborated in (a) with $\phi=180^{\circ}$. However, for $\theta=20^{\circ}$ (i.e., $\theta=\theta_{m}$ for $10^{21} \mathrm{~W} / \mathrm{cm}^{2}$ ) the spectrum of the emitted radiation (b), and corresponding phase (d) is presented. We have also presented the emitted attosecond pulses along $\theta=20^{\circ}$ (c), for the cases when the cluster is centered around $(0,0,-10),(0,0,0)$, and $(0,0,+10)$.

obtain attosecond pulses of duration $\sim 276$ as for laser intensity of $10^{21} \mathrm{~W} / \mathrm{cm}^{2}$. The direction of pulse detection also plays a crucial role here, and in order to obtain a single attosecond pulse of high intensity, we have conducted our study in the direction of maximum power radiation for a given intensity as estimated by Eq. (19).

Earlier in Fig. 2, we already mentioned that the time evolution of the acceleration for a single macroparticle is equivalent to the total acceleration of 100 macroparticles. Does the temporal evolution of acceleration also translate to the temporal and spatial coherence in the emitted attosecond pulses? In order to understand the coherence properties of the emitted signal, we present the angular distribution of the emitted signals, the spectrum and its phase in Fig. 7. In this particular case we have used the laser intensity of $10^{21} \mathrm{~W} / \mathrm{cm}^{2}$, and keeping the computing rigor in mind, the cluster is modeled with just 10 macroparticles while keeping the same charge to mass ratio. It can be observed from Fig. 7(a) that the most energetic pulse is emitted around $\theta=20^{\circ} \pm 0.5^{\circ}$ as estimated by Eq. (19) for the given laser intensity. The spectrum of the radiation as evaluated for $\theta=20^{\circ}$ in Fig. 7(b) is just a scaled down version of the spectrum presented in Fig. 5(d), where we have relied on 100 macroparticles. In Fig. 7(d), we present the phase of the emitted signal around the spectrum peak as presented in Fig. 7(b). It can be seen that those phases are not completely random, which might hint towards the temporal coherence of the collective emitted signal. The effect of spatial translation on the emitted signal is also illustrated in Fig. 7(c), where we have compared the emitted attosecond pulses when cluster is displaced by $z= \pm 10$ (equivalent of $\sim 1000 R_{0}$ ) along the direction of the laser propagation. As expected, it is observed that the duration of the emitted pulse is unchanged which indicates toward good spatial coherence as well.

Furthermore, it should be noted that in experimental scenario, gas pressure, nozzle parameters etc, control the size (diameter) of individual clusters along with the cluster density (number of clusters per unit volume).The laser pulse interacts with an ensemble of clusters, which are typically separated by several hundreds of nanometers [50]. In this context the emission of attosecond bursts of energy from individual clusters can be treated as an independent event.

\section{CONCLUDING REMARKS}

In this work we have studied the laser-cluster interaction using a classical 3D molecular dynamics simulation tool [27]. The objective is to utilize the atomic clusters to produce the harmonics of highly intense laser pulses through Thomson scattering. The effect of laser intensity on the total energy radiated is studied and compared with the expected scaling laws. It has been observed that the numerical simulations accurately produce the scaling law which takes care of the ponderomotive force and the angles at which maximum radiations are emitted [refer to Eq. (19)]. Hence, this setup can be used as a diagnostic tool to directly measure the peak laser intensities. As we have seen in the results presented, relativistic Thomson 
scattering of electrons during laser-cluster interaction can produce harmonics of order as high as $250 \omega_{0}$ for laser intensity $10^{21} \mathrm{~W} / \mathrm{cm}^{2}$. Hence, in case of the $248 \mathrm{~nm}$ laser that we used, the radiation emitted can have wavelength as low as $\sim 10 \AA$, which falls under soft X-rays. Apart from this, we have also calculated the angular distribution of emitted harmonics [refer to Fig. 4] and it is observed that particular harmonics are emitted along specific directions, which in principle is useful to tune the detectors to utilize the radiation of a particular energy [51]. The generation and application of the attosecond pulses is interesting in its own right and in this work, we were able to obtain the attosecond pulses ranging from $\sim 80-276$ as with good spatial and temporal coherence properties, depending on the incident laser intensity. Furthermore, the cluster source gives additional tweaking capabilities to increase the intensity of emitted attosecond pulses by changing the cluster configurations. The cluster species and size actually dictate the number of electrons participating in the harmonic emission and consequently, manipulate the attosecond pulse generation.

In conclusion, we can say that the interaction of high intensity few cycles pulses with atomic clusters can lead to nonlinear relativistic Thomson scattering, leading to emission of radiation which has important diagnostic potential. The use of clusters in this process ensures the production of electrons within the premises of incident intense laser pulse. Also, while this study has been done using an ultrashort and intense $\mathrm{KrF}$ laser pulse, the physics involved in the nonlinear Thomson scattered radiation and the diagnostics associated with it will be functional for intense pulses of longer wavelength as well. The $\mathbf{v} \times \mathbf{B}$ drift is responsible for the intensity dependent changes in the radiated power, which is an important factor in our study having significant diagnostic potential. This drift is present due to the strong magnetic field associated with the intense laser fields and has been reported in studies using NIR $(800 \mathrm{~nm})$ pulses too [40].

In this paper, since we have used very high intensity, few cycles pulse, the cluster dynamics happens almost instantaneously, which is why we have focused on the latter part of the process, i.e., the electron dynamics and radiation spectra. However, the interaction dynamics within the cluster can be studied in terms of the radiation spectra using lower intensity, longer duration pulses. In this case, slow ionization and energetics of the cluster will be reflected in the radiation spectrum of electrons [14]. However, this would be a detailed analysis which we reserve for future work.

\section{ACKNOWLEDGMENTS}

The authors would like to acknowledge the Department of Physics, Birla Institute of Technology and Science, Pilani, Rajasthan, India for the computational support.
A. H. acknowledges the Science and Engineering Research Board, Department of Science and Technology, Government of India for funding the Project No. EMR/ 2016/002675.

[1] F. Gilleron, M. Poirier, T. Blenski, M. Schmidt, and T. Ceccotti, Emissive properties of xenon ions from a laserproduced plasma in the 100-140 Å spectral range: Atomicphysics analysis of the experimental data, J. Appl. Phys. 94, 2086 (2003).

[2] S. Zamith, T. Martchenko, Y. Ni, S. A. Aseyev, H. G. Muller, and M. J. J. Vrakking, Control of the production of highly charged ions in femtosecond-laser cluster fragmentation, Phys. Rev. A 70, 011201 (2004).

[3] T. D. Donnelly, T. Ditmire, K. Neuman, M. D. Perry, and R. W. Falcone, High-Order Harmonic Generation in Atom Clusters, Phys. Rev. Lett. 76, 2472 (1996).

[4] J. Zweiback, T. E. Cowan, R. A. Smith, J. H. Hartley, R. Howell, C. A. Steinke, G. Hays, K. B. Wharton, J. K. Crane, and T. Ditmire, Characterization of Fusion Burn Time in Exploding Deuterium Cluster Plasmas, Phys. Rev. Lett. 85, 3640 (2000).

[5] I. Last and J. Jortner, Nuclear Fusion induced by Coulomb Explosion of Heteronuclear Clusters, Phys. Rev. Lett. 87, 033401 (2001).

[6] W. Bang, H. J. Quevedo, A. C. Bernstein, G. Dyer, Y. S. Ihn, J. Cortez, F. Aymond, E. Gaul, M. E. Donovan, M. Barbui, A. Bonasera, J. B. Natowitz, B. J. Albright, J. C. Fernández, and T. Ditmire, Characterization of deuterium clusters mixed with helium gas for an application in beamtarget-fusion experiments, Phys. Rev. E 90, 063109 (2014).

[7] A. Sharma, High Energy electron and proton acceleration by circularly polarized laser pulse from near critical density hydrogen gas target, Sci. Rep. 8, 2191 (2018).

[8] A. Barna, I. B. Földes, J. Bohus, and S. Szatmári, Active stabilization of the beam pointing of a high- power $\mathrm{KrF}$ laser system, Metrol. Meas. Syst. 22, 165 (2015).

[9] High intentsity laser laboratory, https://www.laserlabeurope.eu/about-us/partners/partners-dp.

[10] J. M. Cole et al., Experimental Evidence of Radiation Reaction in the Collision of a High-Intensity Laser Pulse with a Laser-Wakefield Accelerated Electron Beam, Phys. Rev. X 8, 011020 (2018).

[11] J. K. Koga, S. V. Bulanov, T. Z. Esirkepov, M. Kando, S. S. Bulanov, and A.S. Pirozhkov, Relativisitcally upshifted higher harmonic generation via relativistic flying mirrors, Plasma Phys. Controlled Fusion 60, 074007 (2018).

[12] A. V. Mitrofanov, D. A. Sidorov-Biryukov, M. V. Rozhko, S. V. Ryabchuk, A. A. Voronin, and A. M. Zheltikov, High-order harmonic generation from a solid-surface plasma by relativistic-intensity sub-100-fs mid-infrared pulses, Opt. Lett. 43, 5571 (2018).

[13] F. Mackenroth and A. R. Holkundkar, Determining the duration of an intense laser pulse directly in focus, arXiv: 1712.06898.

[14] V. S. Rastunkov and V. P. Krainov, Mechanisms for second harmonic generation in the interaction of a superintense 
ultrashort laser pulse with cluster plasma, Laser Phys. 17, 625 (2007).

[15] J. D. Jackson, Classical Electrodynamics (Wiley, New York, 1975).

[16] P. Lan, P. Lu, W. Cao, and X. Wang, Nonlinear Thomson scattering in the few-cycle regime, J. Phys. B 40, 403 (2007).

[17] R. Lichters, J. Meyer-ter-Vehn, and A. Pukhov, Short-pulse laser harmonics from oscillating plasma surfaces driven at relativistic intensity, Phys. Plasmas 3, 3425 (1996).

[18] G. M. Petrov, J. Davis, and A. L. Velikovich, Tunable synchrotron radiation from high intensity laser-cluster interaction, J. Phys. B 39, 4617 (2006).

[19] J. Gao, Thomson Scattering from Ultrashort and Ultraintense Laser Pulses, Phys. Rev. Lett. 93, 243001 (2004).

[20] A. V. Balakin, A. V. Borodin, M. S. Dzhidzhoev, V. M. Gorgienko, M. N. Esaulkov, I. A. Zhvaniya, N. A. Kuzechkin, I. A. Ozheredov, A. Y. Sidorov, P. M. Solyankin, and A. P. Shkurinov, Terahertz emission during interaction of ultrashort laser pulses with gas cluster beam, J. Phys. Conf. Ser. 735, 012021 (2016).

[21] F. Dorchies, N. Jourdain, L. Lecherbourg, and P. Renaudin, Comparisons of $\mathrm{x}$-ray sources generated from subpicosecond laser-plasma interaction on clusters and on solid targets, Phys. Rev. E 98, 033212 (2018).

[22] L. M. Chen, W. C. Yan, D. Z. Li, Z. D. Hu, L. Zhang, W. M. Wang, N. Hafz, J. Y. Mao, K. Huang, Y. Ma, J. R. Zhao, J. L. Ma, Y. T. Li, X. Lu, Z. M. Sheng, Z. Y. Wei, J. Gao, and J. Zhang, Bright betatron X-ray radiation from a laser-driven-clustering gas target, Sci. Rep. 3, 1912 (2013).

[23] Q. Zhang, K. Zhao, J. Li, M. Chini, Y. Cheng, Y. Wu, E. Cunningham, and Z. Chang, Suppression of driving laser in high harmonic generation with a microchannel plate, Opt. Lett. 39, 3670 (2014).

[24] F. L. Kien, K. Midorikawa, and A. Suda, Attosecond pulse generation using high harmonics of the $\mathrm{KrF}$ laser driver pulse, J. Appl. Phys. 37, L733 (1998).

[25] H. Nguyen, A. Suda, and K. Midorikawa, Attosecond pulse generation in $\mathrm{He}$ gas with few-cycle $\mathrm{KrF}$ driver pulses, J. Appl. Phys. 38, 6298 (1999).

[26] P. M. Paul, E. S. Toma, P. Breger, G. Mullot, F. Augé, P. Balcou, H. G. Muller, and P. Agostini, Observation of a train of attosecond pulses from high harmonic generation, Science 292, 1689 (2001).

[27] P. Venkat and A. R. Holkundkar, Interaction of xenon clusters with intense sub-cycle laser pulses, Phys. Plasmas 23, 123106 (2016).

[28] A. R. Holkundkar, Parallel implementation of threedimensional molecular dynamic simulation for lasercluster interaction, Phys. Plasmas 20, 113110 (2013).

[29] E. Esarey, P. Sprangle, J. Krall, and A. Ting, IEEE Plasma Sci. 24, 252 (1996).

[30] A. R. Holkundkar, C. Harvey, and M. Marklund, Thomson scattering in high-intensity chirped laser pulses, Phys. Plasmas 22, 103103 (2015).

[31] G. M. Petrov, J. Davis, A. L. Velikovich, P. C. Kepple, A. Dasgupta, R. W. Clark, A. B. Borisov, K. Boyer, and C. K. Rhodes, Modeling of clusters in a strong $248 \mathrm{~nm}$ laser field by a three-dimensional relativistic molecular dynamic model, Phys. Rev. E 71, 036411 (2005).

[32] G. M. Petrov and J. Davis, Modeling of clusters by a molecular dynamics model using a fast tree method, Eur. Phys. J. D 41, 629 (2007).

[33] I. Last and J. Jortner, Scaling procedure for simulations of extreme ionizations and Coulomb explosion of large clusters, Phys. Rev. A 75, 042507 (2007).

[34] G. M. Petrov, J. Davis, A. L. Velikovich, P. Kepple, A. Dasgupta, and R. W. Clark, Dynamics of a Xe cluster plasma produced by an intense ultrashort pulse $\mathrm{KrF}$ laser, Phys. Plasmas 12, 063103 (2005).

[35] M. Ammosov, N. Delone, and V. Krainov, ZhETF 91, 2008 (1986) [JETP 64 (1986)].

[36] W. Lotz, Electron-impact ionization cross-sections and ionization rate coefficients for atoms and ions from hydrogen to calcium, Z. Phys. 216, 241 (1968).

[37] P. Venkat and A. R. Holkundkar, Energetic electron bunch generation by laser interaction with xenon clusters, Phys. Plasmas 25, 103112 (2018).

[38] D. S. Filippychev, Computing the particle paths in an opentrap sharp-point geometry, Comput. Math. Model. 12, 193 (2001).

[39] I. Ghebregziabher, B. A. Shadwick, and D. Umstadter, Spectral bandwidth reduction of Thomson scattered light by pulse chirping, Phys. Rev. Accel. Beams 16, 030705 (2013).

[40] K. Lee, Y. H. Cha, M. S. Shin, B. H. Kim, and D. Kim, Relativistic nonlinear Thomson scattering as attosecond X-ray source, Phys. Rev. E 67, 026502 (2003).

[41] E. Esarey, S. K. Ride, and P. Sprangle, Nonlinear Thomson scattering of intense laser pulses from beams and plasmas, Phys. Rev. E 48, 3003 (1993).

[42] S. Harris, J. Macklin, and T. Hänsch, Atomic scale temporal structure inherent to high-order harmonic generation, Opt. Commun. 100, 487 (1993).

[43] G. Farkas and C. Tóth, Proposal for attosecond light pulse generation using laser induced multiple-harmonic conversion processes in rare gases, Phys. Lett. A 168, 447 (1992).

[44] A. E. Kaplan, Subfemtosecond Pulses in Mode-Locked $2 \pi$ Solitons of the Cascade Stimulated Raman Scattering, Phys. Rev. Lett. 73, 1243 (1994).

[45] P. Lan, P. Lu, and W. Cao, Single attosecond pulse generation by nonlinear Thomson scattering in a tightly focused intense laser beam, Phys. Plasmas 13, 013106 (2006).

[46] W. Luo, H. B. Zhuo, Y. Y. Ma, Y. M. Song, Z. C. Zhu, T. P. Yu, and M. Y. Yu, Attosecond Thomson-scattering $\mathrm{X}$-ray source driven by laser-based electron acceleration, Appl. Phys. Lett. 103, 174103 (2013).

[47] K. Lee, B. H. Kim, and D. Kim, Coherent radiation of relativistic nonlinear Thomson scattering, Phys. Plasmas 12, 043107 (2005).

[48] F. Liu and O. Willi, Ultrashort x-ray pulse generation by nonlinear Thomson scattering of a relativistic electron with an intense circularly polarized laser pulse, Phys. Rev. Accel. Beams 15, 070702 (2012). 
[49] P. Lan, P. Lu, W. Cao, and X. Wang, Attosecond and zeptosecond X-ray pulses via nonlinear Thomson backscattering, Phys. Rev. E 72, 066501 (2005).

[50] M. Aladi, R. Bolla, P. Rácz, and I. Földes, Noble gas clusters and nanoplasmas in high harmonic generation,
Nucl. Instrum. Methods Phys. Res., Sect. B 369, 68 (2016).

[51] Z. Cao, F. Jin, J. Dong, Z. Yang, X. Zhan, Z. Yuan, H. Zhang, S. Jiang, and Y. Ding, Soft X-ray low-pass filter with a squarepore microchannel plate, Opt. Lett. 38, 1509 (2013). 